\title{
On the Global Controllability of Hybrid Systems: Hybrifolds and Fountains. ${ }^{1}$
}

\author{
Ekaterina S. Lemch, Shankar Sastry \\ EECS, University of California at Berkeley \\ 275 Cory Hall 1774 \\ Berkeley, CA, 94720-1774, U.S.A. \\ <lemch,sastry>@eecs.berkeley.edu
}

\author{
Peter E. Caines \\ ECE, McGill University \\ 3480 University Street, Montreal \\ Quebec, H3A 2A7, Canada \\ peterc@cim.mcgill.ca
}

\begin{abstract}
In this paper we investigate the question of the global controllability posed for a class of control hybrid systems. New sufficient conditions for the global controllability are obtained in terms of the so-called hybrid fountains. The main tool for our analysis is the notion of a controlled hybrifold.
\end{abstract}

Keywords: control hybrid systems, global controllability, fountains, controlled hybrifolds.

\section{Introduction}

In this paper we consider systems which have a hybrid nature, in the sense that the dynamics of the system combines continuous and discrete components. We model control hybrid systems as a tuple consisting of a state space, a set of admissible controls, a family of controlled autonomous vector fields assigned to each discrete state, and collections of guards and reset maps.

The main question investigated in the paper is the controllability of control hybrid systems. This issue has been addressed in $[1,5,10,11]$. In particular, in [11], a sufficient condition for controllability of hybrid systems is formulated in terms of so-called arrival sets. In [1], the authors derive a necessary and sufficient algebraic condition for a certain subclass of piecewise affine hybrid systems. In [10], the notion of controllability for hybrid systems is formalized by continuity of system functions.

In this paper new sufficient conditions for the global controllability are obtained in terms of the so called hybrid fountains. The main tool for our analysis is the notion of a hybrifold, which was originally introduced in [12].

The paper is organized as follows. In Section 2, we formally define the class of control hybrid systems $H$

\footnotetext{
${ }^{1}$ The work is supported by DARPA under F33615-98-C-3614 and NSERC grant number OGP 0001329.
}

0-7803-6638-7/00\$10.00 (C) 2000 IEEE under our consideration and specify the standard assumptions on the continuous and discrete parts of the dynamics of $H$. In Section 3, we generalize to control hybrid systems the notions of a hybrid execution and a hybrid flow originally introduced in $[8,9,13]$ for regular hybrid systems. Next we briefly overview the procedure for constructing the so-called hybrifold [12] and define a controlled flow on it. Section 4 relates the global controllability of $H$ to the global controllability of the associated controlled hybrifold. In Section 5, we introduce the notion of a hybrid fountain and provide new sufficient conditions for the global controllability of control hybrid systems. Finally in Section 6, we illustrate the theory developed in the paper on a water tank system example.

\section{Regular Control Hybrid Systems: Standing Assumptions}

We consider control hybrid systems which in this paper are taken to be of the following form.

Definition 1 An $n$-dimensional control hybrid systems $H$ is the 7-tuple $H=\{Q, \mathcal{D}, E, \mathcal{U}, \mathcal{X}, \mathcal{G}, \mathcal{R}\}$, where

$Q=\{1, \cdots, k\}, 1 \leq k<\infty$, is the set of discrete states (which are called control locations);

$\mathcal{D}=\left\{D_{i} ; i \in Q, D_{i} \subset \mathbb{R}^{n}\right\}$ is the collection of domains;

$E \subset Q \times Q$ is a finite set of transition labels (or edges);

$\mathcal{U}$ is the set of admissible control functions taking values in some $U \subset \mathbb{R}^{n_{u}}$;

$\mathcal{X}=\left\{f_{i} ; i \in Q, f_{i}: D_{i} \times U \rightarrow \mathbb{R}^{n}\right\}$ is the collection of autonomous control vector fields assigned to each control location;

$\mathcal{G}=\left\{G_{e} ; e=(i, j) \epsilon E, G_{e} \subset i \times D_{i}\right\}$ is the collection of guards; 
$\mathcal{R}=\left\{R_{e} ; e=(i, j) \epsilon E, R_{e} \subset G_{e} \times j \times D_{j}\right\}$ is the collection of resets.

In this section we briefly overview and generalize to control hybrid systems some of the notions introduced in $[8,9,13]$ for hybrid systems. Henceforth we shall use the following notations:

$D \triangleq \bigcup_{i \in Q} i \times D_{i}$ shall denote the total domain of $H$.

$\tilde{D} \triangleq D-\bigcup_{e \in E}\left(G_{e} \cup R_{e}\left(G_{e}\right)\right)$, in case $R_{e}$ is a map, for all $e \epsilon E$.

As in [12], we shall restrict ourselves to the study of hybrid systems that are subject to the following assumptions.

A1. The control hybrid system $H$ is deterministic and non-blocking, in the sense that for any family of controls $\left\{u_{1}, u_{2}, \cdots, u_{k} ; i \epsilon Q, u_{i} \in \mathcal{U}\right\}$ and any initial condition $t_{0}, x_{0}$, the hybrid system $H_{u} \triangleq\left\{Q, \mathcal{D}, E, \mathcal{U}, \mathcal{X}_{u}, \mathcal{G}, \mathcal{R}\right\}$, where $\mathcal{X}_{u} \leqq\left\{f_{i}\left(\cdot, u_{i}\right)\right\}$, a an infinite execution starting at $\left(t_{0}, x_{0}\right)$ and such execution is unique. The reader is referred to [8] for necessary and sufficient conditions for a hybrid system to be deterministic and non-blocking.

A2. For each $i \epsilon Q, D_{i}$ is assumed to be a non-empty, closed, contractible $n$-dimensional sub-manifold of $\mathbb{R}^{n}$, with a piecewise smooth boundary. No two smooth components of the boundary $\partial D_{i}$ meet at a zero angle.

A3. For each $e=(i, j) \epsilon E$, the guard $G_{e}$ is a closed piecewise smooth $(n-1)$-dimensional sub-manifold of $i \times \partial D_{i}$ with a piecewise smooth (or possibly empty) boundary. $G_{e}$ has a finite number of connected components.

A4. For each $e=(i, j) \in E$, the reset $R_{e}$ is a homeomorphic map from $G_{e}$ onto $R_{e}\left(G_{e}\right)$ which is a subset of $j \times \partial D_{j}$.

A5. For any $e, e^{\prime} \in E, G_{e} \cap R_{e^{\prime}}\left(G_{e^{\prime}}\right)=\emptyset, G_{e} \cap G_{e^{\prime}} \neq$ $\emptyset \Longrightarrow\left(e=e^{\prime}\right)$, and $\left[R_{e}\left(G_{e}\right)\right]^{\circ} \cap\left[R_{e^{\prime}}\left(G_{e^{\prime}}\right)\right]^{\circ} \neq \emptyset \Longrightarrow$ $\left(e=e^{\prime}\right)$. Thus only images of resets are allowed to have intersections and that can happen only at their boundary points.

Furthermore, any $p \in \partial G_{e} \cup \partial\left\{R_{e}\left(G_{e}\right)\right\}$, for some $e \epsilon E$, satisfy some technical conditions specified in [12].

A6. For any $p \epsilon G_{e}, e=(i, j) \epsilon E$, there exists a control $u \in \mathcal{U}$ under which a state $z \epsilon\left(i \times D_{i}\right) \cap \tilde{D}$ can be driven to $p$ (under $u$ ) in such a way that the trajectory does not leave $\left(i \times D_{i}\right) \cap \tilde{D}$.

Similarly, for any $p^{\prime} \epsilon R_{e}\left(G_{e}\right)$, there exists a control $u^{\prime} \epsilon \mathcal{U}$ under which $p^{\prime}$ can be driven to a state $z^{\prime} \epsilon$ $\left(j \times D_{j}\right) \cap \tilde{D}$ (under $u^{\prime}$ ) in such a way that the trajectory does not leave $\left(j \times D_{j}\right) \cap \tilde{D}$.
Next we list the assumptions on the continuous part of the dynamics of $H$.

B1. For each $i \epsilon Q, X_{i} \epsilon C^{r}\left(D_{i} \times U ; \mathbb{R}^{n}\right), r \epsilon$ $\{1,2, \cdots, \infty, \omega\}$, where $C^{\omega}$ denotes the class of analytic functions.

B2. The set of admissible control functions

$$
\mathcal{U}=\mathcal{U}^{s}\left(\mathbb{R} ; \mathbb{R}^{n_{u}}\right), s \in\{1,2, \cdots, \infty\},
$$

is the set of all $\mathbb{R}^{n_{u}}$-valued bounded piecewise $C^{s}\left(\mathbb{R} ; \mathbb{R}^{n_{u}}\right)$ functions of time with limits from the right; Hence any $u \in \mathcal{U}$, defined on some $\left[T_{1}, T_{2}\right), T_{2}<\infty$, is $C^{s}$ on $\left[T_{1}, T_{2}\right)$ with the exception of a finite number of points.

For the results formulated in this paper we shall need $r=1, s=1$.

Definition 2 A control hybrid system satisfying assumptions A1-A6 and B1-B2 is called a regular control hybrid system.

\section{Control Hybrifold}

Let $H$ be a regular control hybrid system. We shall use the standard notion of a forward hybrid time trajectory $\tau=\left\{\left[\tau_{i}, \tau_{i+1}\right) ; \tau_{i}<\tau_{i+1}\right\}$, as defined in $[8,9,12,13]$. We shall use he symbol $N(\tau)$ to denote the size of the time trajectory and the symbol $\langle\tau\rangle$ to denote the set $\{1,2, \cdots, N(\tau)\}$.

As follows from the assumption $B 2$, for'any control $u \epsilon$ $\mathcal{U}$ defined on some $\left[T_{1}, T_{2}\right), T_{2}<\infty$, there exists a finite hybrid time trajectory $\tau \epsilon \Im$ such that $u$ is $C^{q}$ on each $I_{i} \epsilon \tau, i \epsilon\langle\tau\rangle$, and $\tau_{1}=T_{1}, \tau_{N(\tau)+1}=T_{2}$. We shall say that such $\tau$ is the hybrid time trajectory of $u$ and denote it as $\tau(u)$.

\section{Definition 3 (Forward Control Execution)}

For any $u \in \mathcal{U}$, defined on $\left[T_{1}, T_{2}\right), T_{2}<\infty$, and $p \in D$, we define a (forward) control execution starting at $p$ as a triple $\chi=(\tau, q, \phi)$, where $\tau \epsilon \Im$ is a refinement of $\tau(u) ; q:\langle\tau\rangle \rightarrow Q$ is a map; and $\phi=\left\{\phi_{j}:\left[\tau_{j} ; \tau_{j+1}\right) \rightarrow D_{q(j)} ; j \epsilon\langle\tau\rangle\right\}$ is a collection of continuously differentiable maps such that $\phi_{1}\left(\tau_{1}\right)=p$, and $\dot{\phi}_{j}(t)=X_{q(j)}\left(\phi_{j}(t), u_{j}\right)$.

Furthermore, for all $j \epsilon\langle\tau\rangle, j \neq N$, we must have $(q(j), q(j+1)) \epsilon E, a_{j} \triangleq\left\{\lim _{t \rightarrow \tau_{j+1}} \phi_{j}(t)\right\} \epsilon G_{(q(j), q(j+1))}$, and $R_{(q(j), q(j+1))}\left(a_{j}\right)=\phi_{j+1}\left(\tau_{j+1}\right)$.

Lemma 1 Let $H$ be a regular control hybrid system. For any $u^{*} \epsilon \mathcal{U}$ defined on some $\left[T_{1}, T_{2}\right), T_{2}<\infty$, and 
any $p \epsilon D$, there exists a unique control execution of $H$.

In [12], a set $M_{H}$ (called the hybrifold) is constructed from a hybrid system $H$. In this section we briefly overview this procedure and define the controlled hybrid flow on $M_{H}$. The basic idea in the construction of the hybrifold is to glue together each guard to the image of the corresponding reset map by identifying any state $p \in G_{e}$, where $e \in E$, with the corresponding image $R_{e}(p)$. So an equivalence relation $\sim$ on $D$ is generated by $p \sim R_{e}(p)$, for all $e \epsilon E$ and $p \epsilon G_{e}$. This relation gives rise the quotient space $M_{H}=D / \sim$, where each equivalence class is collapsed to a point. The set $M_{H}$ is called the hybrifold of $H$.

Let $\pi$ be the natural projection map $\pi: D \rightarrow M_{H}$ which assigns to each $p$ its equivalence class. We put the quotient topology on $M_{H}$, i.e. the smallest topology in which $V \subset M_{H}$ is open if and only if $\pi^{-1}(V) \subset D$ is open (in the relative topology of $D$ ).

As has been proven in [12], $M_{H}$ is a topological $n$ manifold with boundary. Thus we shall assume, without loss of generality, that $M_{H}$ is embedded in $\mathbb{R}^{m}$, for some $m, n \leq m<\infty$. Henceforth we shall deal not with the original domains $D_{i}$ but rather with the hybrifold $M_{H}$.

Remark 1 Take an arbitrary $x \in M_{H}$ and consider its inverse image under $\pi$. As follows from the assumption A5, there are two possible cases: (i) $\pi^{-1}(x)$ is a singleton, i.e. $p=\pi^{-1}(x) \epsilon \tilde{D}$; and (ii) $\pi^{-1}(x)=$ $\left\{z_{1}, \cdots, z_{s}, p\right\}$, where each $z_{i}, 1 \leq i \leq s<\infty$, lies on the boundary of a guard $G_{e_{i}}$, for some $e_{i} \epsilon E$, and $p=R_{e_{1}}\left(z_{1}\right)=\cdots=R_{e_{s}}\left(z_{s}\right)$. In both cases we shall take $p$ as the canonical representative of the equivalence class $\pi^{-1}(x)$.

Definition 4 (Hybrid Control Flow) Take an arbitrary control $u \in \mathcal{U}$ defined on some $\left[T_{1}, T_{2}\right), T_{2}<\infty$, and an arbitrary state $x \epsilon M_{H}$. Let $p \epsilon D$ be the canonical representative of the equivalence class $\pi^{-1}(x)$.

As follows from Lemma 1, there exists a unique control execution $\chi=\{\tau, q, \phi\}$ of $H$ starting at $p$.

We shall use the symbol $\Psi^{H}(t, x, u), t \in\left[T_{1}, T_{2}\right)$, to denote the controlled hybrid flow on $M_{H}$ :

$\Psi^{H}(t, x, u) \triangleq \pi\left(\phi_{i}(t)\right)$, for any $i \cdot \epsilon\langle\tau\rangle$ and $t \epsilon\left[\tau_{i}, \tau_{i+1}\right)$.

$M_{H}$ with the controlled flow $\Psi^{H}$ defined on it shall be called the controlled hybrifold of $H$.

Lemma 2 For any control $u$, the controlled hybrid flow $\Psi^{H} \cdot(\cdot, x, u)$ is continuous on $M_{H}$ with respect to the argument $t$.

\section{Global Controllability of Hybrid Systems}

Let $H$ be an arbitrary regular control hybrid system and $M_{H}$ its controlled hybrifold. In this section we relate the global controllability of the total domain $D$ of $H$ with the global controllability of $M_{H}$.

\section{Definition 5 (Accessible sets of $H$ )}

We shall say that a state $p^{\prime} \epsilon D$ is accessible from $p$ in $D$ (with respect to $V \subset D$ ) if there exists a control $u \in \mathcal{U}$, defined on some $\left[T_{1}, T_{2}\right), T_{2}<\infty$, and its control execution $\chi=(\tau, q, \phi)$ of $H$ starting at $p$ such that

(i) $\phi_{N(\tau)}(T)=p^{\prime}$, for some $T \epsilon\left[\tau_{N(\tau)} ; \tau_{N(\tau)+1}\right)$; and

(ii) for any $j \epsilon\langle\tau\rangle$ and $t \epsilon\left[\tau_{j} ; \tau_{j+1}\right), \phi_{j}(t) \epsilon V$.

The set of all states in $D$ accessible from $p$ (with respect to $V$ ) shall be denoted by $A_{D}^{V}(p)$. In the case $V=D$, we shall write $A_{D}(p)$.

Thus we assumed that an accessible state $p^{\prime}$ can be reached from $p$ in finite time using a finite number of switching (or jumps) between control locations.

Remark 2 We observe that, as follows from Definition $3, R_{e}(p) \epsilon A_{D}(p)$, for any state $p \in G_{e}, e \epsilon E$.

Similarly, we can define the accessible states using the dynamics of the controlled hybrifold $M_{H}$.

Definition 6 (Accessible sets of $M_{H}$ )

Let $x \in M_{H} \subset \mathbb{R}^{m}$. We shall say that a state $x^{\prime} \in M_{H}$ is accessible from $x$ (with respect to $V \subset M_{H}$ ) if there exists a control $u \in \mathcal{U}$ defined on some $\left[T_{1}, T_{2}\right), T_{2}<\infty$, such that

(i) $x^{\prime}=\Psi^{H}(T, x, u)$, for some $T \epsilon\left[T_{1}, T_{2}\right)$; and

(ii) for any $T_{1} \leq t \leq T, \Psi^{H}(t, x, u) \in V$.

The set of all states in $M_{H}$ accessible from $x$ (with respect to $V$ ) shall be denoted by $A^{V}(x)$. In the case $V=M_{H}$, we shall write $A(x)$.

The set of all states co-accessible to $p$ (to $x$ ), with respect to $V \subset D$ (with respect to $V \subset M_{H}$ ), in $H$ (in $\left.M_{H}\right)$ is defined dually and shall be denoted as $C A_{D}^{V}(p)$ (as $C A^{W}(x)$ ).

Remark 3 We observe that for any $p \in D$ and any neighborhood $V$ of $p$ in $D$, we have

$$
\pi\left(A_{D}^{V}(p)\right) \subset A^{\pi(V)}(\pi(p)),
$$


where $\pi: D \longrightarrow M_{H}$ is the natural projection map. This is because any orbit in $D$ is projected by $\pi$ onto an orbit in $M_{H}$.

In the particular case $V=M_{H}$, we have the following result.

\section{Theorem 1}

(i) $\forall p, p^{\prime} \in D \quad\left(p \sim p^{\prime}\right) \Longrightarrow A_{D}(p)-\left\{p, p^{\prime}\right\}=A_{D}\left(p^{\prime}\right)-$ $\left\{p, p^{\prime}\right\}$.

Hence, subject to this condition, $A_{D}(p) \cup A_{D}\left(p^{\prime}\right)=$ $A_{D}(p) \cup\left\{p, p^{\prime}\right\}$.

(ii) The relation $\sim$ is a bisimulation on $D \times D$, in the sense that if a state $p$ can be driven to some $y$, then any $p^{\prime}, p^{\prime} \sim p$, can be driven to some $y^{\prime}$ such that $y^{\prime} \sim y$.

(iii) $\forall p \in D \quad \pi\left(A_{D}(p)\right)=A(\pi(p))$.

Definition 7 We say that a set $D_{1} \subset D$ is controllable with respect to $D_{2} \subset D$ for the control hybrid system $H$ if $A_{D}^{D_{2}}(p)=D_{1}$, for all $p \in D_{1}$.

In the particular case when $D_{1}=D, D_{2}=D$, and $A_{D}(p)=D$, for all $p \in D$, we shall say that the total domain $D$ is globally controllable for $H$.

Similarly, we shall say that a set $C_{1} \subset M_{H}$ is controllable with respect to $C_{2} \subset M_{H}$ if $A^{C_{2}}(x)=C_{1}$, for all $x \in C_{1} . M_{H}$ is globally controllable if $A(x)=M_{H}$, for all $x \in M_{H}$.

Theorem 2 Let $H$ be a regular control hybrid system. Then the total domain $D$ is globally controllable if and only if the associated hybrifold $M_{H}$ is globally controllable.

\section{Proof:}

$\Longrightarrow$ Let $D$ be globally controllable. Then, using Remark 3 , we obtain for any $x \in M_{H}$,

$M_{H}=\pi(D)=\pi\left(A_{D}(p)\right) \subset A^{\pi(D)}(\pi(p))=A(x) \subset M_{H}$

where $p$ is an arbitrary point in the set $\pi^{-1}(x) \subset D$ Hence $A(x)=M_{H}$, for any $x \in M_{H}$, and $M_{H}$ is globally controllable.

$\Longleftarrow$ Conversely, let $M_{H}$ be globally controllable. Take any $p, p^{\prime} \in D$. Each of them could lie in any of the sets

$$
\left\{G_{e}, R_{e^{\prime}}\left(G_{e^{\prime}}\right), \tilde{D} ; e, e^{\prime} \epsilon E\right\} .
$$

Consider, for instance, the case when $p \epsilon G_{e}$ and $p^{\prime} \epsilon$ $R_{e^{\prime}}\left(G_{e^{\prime}}\right)$, for some $e=(i, j), e^{\prime}=\left(i^{\prime}, j^{\prime}\right) \epsilon E$. Then define $y=R_{e}(p)$ and $y^{\prime} \in G_{e^{\prime}}$ such that $R_{e^{\prime}}\left(y^{\prime}\right)=p^{\prime}$.
By the assumption A6, the guard and reset conditions are non-vacuous, in the sense that there are states $z \epsilon$ $\left(j \times D_{j}\right) \cap \tilde{D}$ and $z^{\prime} \epsilon\left(i^{\prime} \times D_{i^{\prime}}\right) \cap \tilde{D}$ such that $z$ is accessible from $y$ and $z^{\prime}$ is co-accessible to $y^{\prime}$. Next note, that since $z, z^{\prime} \epsilon \tilde{D}$ and $\pi$ is 1 to 1 on $\tilde{D}$, from the existence of an orbit connecting $\pi(z)$ to $\pi\left(z^{\prime}\right)$ in $M_{H}$ follows the existence of a control execution that drives $z$ to $z^{\prime}$. Finally, combining all the accessibility relations for $p, y, z, z^{\prime}, y^{\prime}, p^{\prime}$ we conclude that $p^{\prime} \in A_{D}(p)$.

The rest of the cases can be considered in an analogous manner. Thus $A_{D}(p)=D$, for any $p \in D$, and $D$ is globally controllable.

The above result allows us to use the hybrifold and the continuous controlled hybrid flow defined on it in order to study the global controllability of the original control hybrid system. The advantage of this approach is in the fact that the controllability results formulated for differential control systems acting on subsets or submanifolds of $\mathbb{R}^{n}$ can be transformed to control hybrid systems. This shall be demonstrated in the next section.

\section{Hybrid Fountains}

In this section we introduce the notion of a hybrid fountain which we shall use as the main hypothesis in our controllability result. Henceforth the symbol $B_{\delta}(x)$, where $x \in M_{H}, 0<\delta \in \mathbb{R}^{1}$, shall denote the $m$ dimensional ball with the center $x$ and the radius $\delta$. The sets $A^{B_{\delta}(p)}(p)$ and $C A^{B_{\delta}(p)}(p)$ shall be denoted as $A^{\delta}(p)$ and $C A^{\delta}(p)$, respectively.

Definition $8 \mathrm{~A}$ state $x \in M_{H}$ is called a hybrid fountain if

$$
\begin{aligned}
& \exists \mu>0 \forall \delta, 0<\delta<\mu, A^{\delta}(x)-\{x\} \text { and } C A^{\delta}(x)-\{x\} \\
& \text { are non-empty, open sets. }
\end{aligned}
$$

If the function $\rho \triangleq \sup \{\mu$; such that (2) holds $\}$ is continuous at $x$, we shall say that $x$ is a continuous hybrid fountain. If $\rho$ is unbounded at $x$ we consider it to be continuous at $x$.

We note that the continuous fountain property does not depend on the particular way that $M_{H}$ is embedded in $\Re^{n}$.

The reader is referred to $[2,3,6]$ for applications of the fountain condition to the study of ordinary differential systems acting on subsets of $\mathbb{R}^{n}$. See also [7], where a set of algebraic conditions for verification of the fountain property is presented, and [4], where applications to hierarchical hybrid control theory are outlined. 
Henceforth we shall use the term controlled closed orbit in the sense of controlled loop.

Theorem 3 Let each $\approx \epsilon M_{H}$ be a continuous hybrid fountain and let for each $x \in M_{H}$ there exist a control $u \in \mathcal{U}$ such that $x$ lies on a nontrivial (controlled under $u$ ) closed orbit in $M_{H}$. Then each connected component of $\left[M_{H}\right]^{\circ}$ is controllable with respect to $M_{H}$.

Proof: Let $C$ denote one of (the finite number of) the connected components of $\left[M_{H}\right]^{\circ}$. For any two states $x, x^{\prime}$ in $C$ we define a relation $\sim_{o}$ in such a way that $x \sim_{o} x^{\prime}$ if and only if there exists a (controlled) nontrivial closed orbit in $M_{H}$ passing through both $x$ and $x^{\prime}$, i.e. there exists a control $u$ defined on some $\left[T_{1}, T_{2}\right)$, $T_{2}<\infty$, such that

(i) $\exists T, T_{1}<T<T_{2}, \quad \Psi\left(T_{1}, x, u\right)=\Psi(T, x, u)$; and

(ii) $\exists \bar{t}, T_{1}<\bar{t} \leq T, \quad \Psi(\bar{t}, x, u)=x^{\prime}$.

Clearly, the relation $\sim_{o}$ is reflexive (since each state in $M_{H}$ lies on a nontrivial orbit), symmetric and transitive. Hence there exists a partition of $C$ on the equivalent classes of $\sim_{o}$. Let $[x]$, for an arbitrary $x \in C$, denote the equivalent class containing $x$. We claim that $[x]$ is an open subset in $C$.

Indeed, take any $z \in[x]$. Let $u$ and $0 \leq t<\infty$ be such that $z=\Psi(t, x, u)$. Define $a=\Psi(t-\Delta, x, u)$ and $b=\Psi(t+\Delta, x, u), \Delta>0$. Then, since $a$ and $b$ are hybrid fountains, the sets $A^{\delta}(a)-\{a\}$ and $C A^{\delta}(b)-\{b\}$ are open, for sufficiently small $\delta>0$. Choose $\Delta$ so small that $z \in A^{\delta}(a)$ and $z \in C A^{\delta}(b)$ (this is possible since $a, b$ are continuous hybrid fountains). Then there exists an open neighborhood $N(z)$ of $z$ which lie in the intersection $\left(A^{\delta}(a)-\{a\}\right) \cap\left(C A^{\delta}(b)-\{b\}\right)$. Each state $z^{\prime} \epsilon N(z)$ is accessible from $a$ and co-accessible to $b$. Moreover, since $a, b \epsilon[x]$, we conclude that $z^{\prime}$ lies on a non-trivial orbit passing through $x$. This is true for all $z^{\prime} \in N(z)$, hence $N(z) \subset[x]$ and $[x]$ is open, as claimed.

For any $x, x^{\prime} \in C$ we have $[x] \cap\left[x^{\prime}\right] \neq \emptyset \Longrightarrow[x]=\left[x^{\prime}\right]$, so any two equivalent classes are either disjoint or coincide. Since $C$ is connected, $C=[x]$. In other words, any two states in $C$ lie on a nontrivial controlled orbit in $M_{H}$ and hence, $C$ is controllable with respect to $M_{H}$.

Theorem 4 Assume that the hybrifold $M_{H}$ is connected and the conditions of Theorem 3 are satisfied. Then $M_{H}$ is globally controllable.

Consider the directed graph $\Gamma$ of $H$ which has vertices $Q$ and edges $E$. We can treat it as a finite state machine, by defining the transition function $\Phi: Q \rightarrow Q$ in such a way that for any $i, j \in Q, \Phi(i)=j$ if and only if $(i, j) \epsilon E$ or $i=j$.

Theorem 5 Assume that the conditions of Theorem 3 are satisfied. Then $M_{H}$ is globally controllable if and only if the graph $\Gamma=\{Q, E\}$ is controllable as a finite state machine.

\section{Proof:}

$\Longrightarrow$ Assume that $M_{H}$ is globally controllable. Then for any $i, j \in Q, i \neq j$, take some states $p \in D_{i}$ and $p^{\prime} \in D_{j}$. There exists a trajectory $\psi$ from $p$ to $p^{\prime}$ in $M_{H}$. Let the sequence $i=r_{1}, r_{2}, \cdots, r_{\ell}=j, \ell>1$, be such that $\psi$ switches consecutively from the domain $D_{r_{s}}$ to the domain $D_{r_{s+1}}$, where $s=1,2, \cdots, \ell-1$, using the corresponding guards and the images of the reset maps. Hence each consecutive pair $\left(r_{s}, r_{s+1}\right)$ belongs to $E$ and hence, there exists a trajectory from the state $i$ to the state $j$ in the graph $\Gamma$. Since this holds for an arbitrary pair $(i, j) \in Q$, we conclude that $\Gamma$ is controllable as a finite state machine.

$\Longleftarrow$ Conversely, assume that $\Gamma$ is controllable as a finite state machine. Then for any two states $p, p^{\prime} \in D$ take $i$ and $j$ such that $p \in D_{i}$ and $p^{\prime} \in D_{j}$. If $i \neq j$, find a trajectory $i=r_{1}, r_{2}, \cdots, r_{\ell}=j, \ell>1$, in the graph $\Gamma$. Since each consecutive pair $\left(r_{s}, r_{s+1}\right)$ belongs to $E$, there exists a guard $G_{\left(r_{s}, r_{s+1}\right)}$ in the domain $D_{r_{s}}$ which is identified with the image of the reset map $R_{\left(r_{s}, r_{s+1}\right)}$ in the domain $D_{r_{s+1}}$. Hence the domains $D_{r_{s}}$ and $D_{r_{s+1}}$, and thus $D_{i}$ and $D_{j}$, lie in one connected component of $M_{H}$. This can be shown for all $i, j \in Q$. Hence $M_{H}$ is connected and, as follows from Theorem $4, M_{H}$ is globally controllable.

Remark 4 In conclusion we note that it is entirely feasible to generalized the presented results to hybrid systems with autonomous and controlled switchings.

\section{Water Tank System}

To illustrate the controllability result obtained in the previous section, we consider a system consisting of two water tanks. The water can be added to the system at some rate $w>0$ (where we treat the parameter $w$ as control) via tank 1 or tank 2 . In addition to that, the water is removed from tank $i, i=1,2$, at some constant rate $v_{i}>0$. We model the system as a control hybrid system and distinguish two control locations :

$$
\begin{aligned}
& q=1:\left\{\dot{x}_{1}=w-v_{1}, \dot{x}_{2}=-v_{2},\left(x_{1}, x_{2}\right) \in D_{1},\right. \\
& q=2:\left\{\dot{x}_{1}=-v_{1}, \dot{x}_{2}=w-v_{2},\left(x_{1}, x_{2}\right) \in D_{2},\right.
\end{aligned}
$$

where $x_{1}, x_{2}$ denote the levels of water in the tanks 1 and 2 , respectively; $D_{1} \underline{\Delta}\left\{\left[l_{1}, \infty\right) \times\left[l_{2}, \infty\right)\right\}$ and $D_{2} \triangleq\left\{\left[l_{1}, \infty\right) \times\left[l_{2}, \infty\right)\right\}$. 


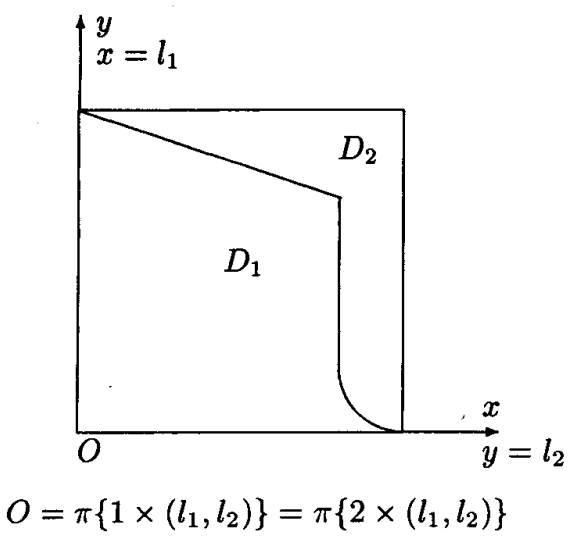

Figure 1: Hybrifold $M_{H}$

The class of control functions is taken to be the set of all piece-wise constant functions of time taking values in the set $U=\left\{w ; w \geq \max \left\{v_{1}, v_{2}\right\}\right\} \subset \mathbb{R}$.

The guards are specified as $G_{(1,2)}=1 \times\left\{\left(x_{1}, l_{2}\right) \in D_{1}\right\}$, and $G_{(2,1)}=2 \times\left\{\left(l_{1}, x_{2}\right) \in D_{2}\right\}$. The resets are defined in such a way that when hitting a guard in one domain the system switches to the other control location, without changing the continuous part of the state, i.e. $R_{(1,2)}\left(1 ; x_{1}, l_{2}\right)=\left(2 ; x_{1}, l_{2}\right)$ and $R_{(2,1)}\left(2 ; l_{1}, x_{2}\right)=$ $\left(1 ; l_{1}, x_{2}\right)$.

The control objective for the system could be formulated in terms of reachability of a final state or a region from an initial state (by means of choosing an appropriate control $w$ ) while keeping the levels of water in the tanks above the levels $x_{1}=l_{1}, x_{2}=l_{2}$ (by means of the defined above guards and resets).

To construct the associated with this system hybrifold we identify (via the identity reset maps) the $x$ and $y$ coordinate axes of $D_{1}$ with the $x$ and $y$ axes of $D_{2}$, respectively. The hybrifold $M_{H}$ is shown in Figure 1.

Remark 5 We note that the only two states in the total domain $D=\left(1 \times D_{1}\right) \cup\left(2 \times D_{2}\right)$ which do not satisfy the assumptions listed in Section 2, (namely A5) are $o_{1} \Delta\left(1 ; l_{1}, l_{2}\right)$ and $o_{2} \Delta\left(2 ; l_{1}, l_{2}\right)$. These are the states that admit the so-called $Z$ eno behavior. For more information on Zeno executions and Zeno states see [12, 13]. We omit these two states and their image $O \Delta$ $\pi\left(o_{1}\right)=\pi\left(o_{2}\right)$ from our consideration. This will not influence our conclusion about controllability, because any control execution in $D$ with the initial condition at $o_{1}$ or $o_{2}$ does not pass through any state in $D-\left\{o_{1}, o_{2}\right\}$.

It can be easily verified that each state $p^{0} \epsilon D^{\circ}$ is a hybrid fountain. Furthermore, each $p \in\left(D-\left\{o_{1}, o_{2}\right\}\right)$ lies on a non-trivial closed control orbit. Applying Theorem 3 , we conclude that $M_{H}-\{O\}$ is controllable and hence, by Theorem 2 and Remark $5, D-\left\{o_{1}, o_{2}\right\}$ is controllable.

\section{References}

[1] A.Bemporad, G. Ferrari-Trecate, and R. Morari. Observability and controllability of piecewise affine and hybrid systems. In Proceedings of the 38th IEEE Control Systems Society Conference on Decision and Control, pages 39663971, Phoenix, AZ., Phoenix, AZ, 1999.

[2] P.E. Caines and E.S. Lemch. On the global controllability of Hamiltonian and other nonlinear systems: fountains and recurrence. In Proceedings of the 37th IEEE Control Systems Society Conference on Decision and Control, pages 3575-3580, Tampa, FL, 1998.

[3] P.E. Caines and E.S. Lemch. On the global controllability of nonlinear systems: fountains, recurrence, and application to Hamiltonian systems. Submitted to SIAM J. on Control and Optimization, 2000.

[4] P.E. Caines and E.S. Lemch. Hierarchical hybrid systems: geometry, controllability and applications to air traffic control. Proceedings of 14th World IFAC, volume E, Beijing, China, July, 1999.

[5] J. Ezzine and A.H. Haddad. Controllability and observability of hybrid systems. Int. J. Control, 49(6):20452055, 1989.

[6] E.S. Lemch. Nonlinear and hierarchical hybrid control systems. Ph.D. thesis, McGill University, Canada, August, 1999.

[7] E.S. Lemch and P.E. Caines. On the global controllability of nonlinear systems: algebraic conditions for the existence of fountains. Fourteenth International Symposium on Mathematical Theory of Networks and Systems, June, 19-23, 2000.

[8] J. Lygeros, K.H. Johansson, S. Sastry, and M. Egerstedt. On the existence of executions of hybrid automata. In Proceedings of the 38th IEEE Control Systems Society Conference on Decision and Control. Phoenix, AZ, Phoenix, AZ, 1999.

[9] J. Lygeros, C. Tomlin, and S. Sastry. Controllers for reachability specifications for hybrid systems. Automatica, 35(3), March, 1999.

[10] A. Nerode and W. Kohn. Models for hybrid systems: automata topologies, controllability, observability. Hybrid Systems, pages 317-356, Berlin, Germany, 10-12 October, 1991.

[11] J.H. Schuppen. A sufficient condition for controllability of a class of hybrid systems. Hybrid Systems: Computation and Control, First International Workshop, Berkeley, CA, April, 1998.

[12] S.N. Simić, K.H. Johansson, S. Sastry, and J. Lygeros. Towards a geometric theory of hybrid systems. Hybrid Systems: Computation and Control, Third International Workshop, Pittsburgh, PA, 2000.

[13] J. Zhang, K.H. Johansson, J. Lygeros, and S. Sastry. Dynamical systems revisited: Hybrid systems with zeno executions. Hybrid Systems: Computation and Control, Third International Workshop, Pittsburgh, PA, 2000. 